\title{
STUDIES ON ALLOYING ELEMENT PARTITIONING IN DMS4 NICKEL BASE SUPERALLOY USING MONTE CARLO SIMULATIONS AND 3D ATOM PROBE
}

\author{
R. Balamuralikrishnan, R. Sankarasubramanian, Mohan P. Pathak, K. Muraleedharan, N. Das \\ Defence Metallurgical Research Laboratory, Kanchanbagh P.O., Hyderabad - 500 058, INDIA
}

Keywords: Nickel base superalloys, Monte Carlo simulation, atom probe field ion microscopy

\begin{abstract}
DMS4 is a third generation single crystal superalloy developed by Defence Metallurgical Research Laboratory (DMRL). Recent efforts have been directed at a thorough characterization of the microstructure to understand the structure-processing-property correlations in this alloy. An important component of this work is to investigate the role of composition on the microstructure. In this paper, we present some results from Monte Carlo simulations on alloying element partitioning in DMS4 as well as model ternary systems. It is found that while the available interatomic potentials are accurate enough to predict partitioning in ternary systems, they appear to be unsuccessful in predicting the structure of multi-component alloys. This conclusion is based on comparison with our own experiments using the $3 \mathrm{D}$ atom probe as well as from data available in the literature on similar alloys.
\end{abstract}

\section{Introduction}

DMS4 is a third generation single crystal nickel-base superalloy developed by the Defence Metallurgical Research Laboratory [1, 2]. This alloy offers more than $80^{\circ} \mathrm{C}$ metal temperature advantage over the first generation single crystal superalloy CMSX-2 and about $8^{\circ} \mathrm{C}$ advantage over modern third generation alloys such as CMSX10. DMS4 has a nominal composition (in weight \%) of: 67Ni-2.4Cr-4Co-5.5W-6.5Re-9Ta-0.1Hf-0.3Nb-5.2Al. The alloy has a density of $9.08 \mathrm{~g} / \mathrm{cc}$ and a total refractory content of $21.4 \mathrm{wt}$. $\%$ including $6.5 \mathrm{wt}$ \% Re to provide improved creep resistance. Mo and Ti have been completely eliminated in order to ensure adequate phase stability. The desired microstructure essentially consists of a homogenous distribution of cuboidal $\gamma^{\prime}$ (crystal structure: $\mathrm{L1}_{2}$ ) particles dispersed in the $\gamma$ (disordered fcc) matrix. This rather uniform structure, with a $\gamma^{\prime}$ size of $\sim 450-500 \mathrm{~nm}$, has been realized through a fairly complex heat treatment cycle that consists of multi-step solutionizing (between $1315^{\circ} \mathrm{C}$ and $1360^{\circ} \mathrm{C}$ ) and multi-step aging treatments (between $1160^{\circ} \mathrm{C}$ and $870^{\circ} \mathrm{C}$ ) [1]. In order to optimize composition and/or processing steps for achieving optimal properties, it is important to understand the partitioning behavior of different alloying elements between $\gamma$ and $\gamma^{\prime}$. This requires the determination of the composition of the two phases. Additional insight might be obtained by determining the sublattice occupancy within the $\gamma^{\prime}$ phase. We have employed both computational (Monte Carlo simulations) and experimental (3D atom probe) techniques to investigate alloying element partitioning.

The output from Monte Carlo simulations for equilibrium microstructures is in the form of a dataset containing the $3 \mathrm{D}$ $(\mathrm{x}, \mathrm{y}, \mathrm{z})$ positions of different atoms in the system. Thus, the first step in the analysis is the identification of phases that is essential before any quantification can be attempted. Phase identification is a non-trivial process, especially in the case of multi-component systems. A prior understanding of the partitioning behavior of the different alloying elements, at least qualitatively, is essential to interpret the complex simulated equilibrium microstructure of multi-component alloys such as DMS4. In this paper, we present some results from Monte Carlo simulations on multi-component as well as model ternary systems. We highlight the challenges regarding phase identification and quantification in these systems and describe our attempts to overcome some of the challenges. We also present atom probe results on alloying element partitioning in fully aged DMS4 alloy and compare with values obtained from our simulations.

\section{Computational Methodology and Experimental Procedure}

A Monte Carlo code has been developed [3] to handle multicomponent systems based on a rigid 3D fcc lattice. Interatomic interactions are described through Lennard-Jones type (8-4) pair potentials [4, 5]. A system size of $64 \times 64 \times 64 \mathrm{fcc}$ unit cells, corresponding to more than a million atoms, has been employed, and periodic boundary conditions have been used. Initially, atoms are distributed randomly at the lattice sites conforming to the desired alloy composition. Equilibrium microstructure is achieved through a series of exchange of nearest neighbor atoms (Kawasaki dynamics) [6] with a probability, p, given by

$$
\mathrm{p}=\exp (-\Delta \mathrm{E} / \mathrm{kT}) /(1+\exp (-\Delta \mathrm{E} / \mathrm{kT}))
$$

where $\mathrm{T}$ is the system temperature, $\mathrm{k}$ is Boltzmann constant and $\Delta \mathrm{E}$ is the change in system energy due to the exchange. The energy of the system is calculated by direct sum of pairwise interatomic interactions up to the third nearest neighbor (3NN) atoms. One Monte Carlo step (MCS) corresponds to as many number of attempted atom exchanges as the number of atoms in the system. The system is assumed to have reached equilibrium when the change in system energy between successive MC steps becomes negligibly small. Equilibrium is ensured by running the simulations up to at least 20,000 MCS. All simulations have been performed at $1273 \mathrm{~K}\left(1000^{\circ} \mathrm{C}\right)$, chosen to represent a realistic aging temperature. AtomEye [7], a freeware program, was used for visualizing the resultant simulated microstructures.

The atom probe specimens were prepared by a standard two-stage electro-polishing procedure as described by Miller and Smith [8]. Initially, $15-20 \mathrm{~mm}$ long blanks with $0.3-0.5 \mathrm{~mm}$ diameter were cut from fully heat-treated blanks using wire EDM. In the first stage, a solution of $25 \%$ perchloric acid in glacial acetic acid was used at $5-15 \mathrm{~V}$ DC to thin down the shank diameter of the blanks and subsequently to obtain a rough conical shaped tip. In the second stage, the final tip was formed by pulse micro-polishing, initially using a solution of $6 \%$ perchloric acid in glacial acetic acid, and subsequently using $2 \%$ perchloric acid in 2-butoxy ethanol at 5$20 \mathrm{~V}$ DC. Atom probe experiments were carried out employing a three-dimensional atom probe with field ion microscope (3DAP 
FIM) instrument manufactured by Oxford nanoScience Ltd., Milton Keynes, UK (presently a subsidiary of Imago, Wisconsin, USA). Field ion images were recorded at a specimen temperature of $70 \mathrm{~K}$ with neon as the imaging gas at $\sim 2.5 \times 10^{-5} \mathrm{mbar}$ pressure. Once a clean field ion image was obtained, the region of interest was chosen and positioned for atom probe data acquisition which was performed at pressures below $8 \times 10^{-10}$ mbar using a $15 \%$ pulse fraction. Data reconstruction, analysis and visualization were performed using the PoSAP software supplied with the instrument.

\section{Results and Discussion}

Initially, a computational system with composition (in atomic \%) 73.04Ni-2.95Cr-4.34Co-1.91W-2.23Re-3.18Ta-12.33Al was successfully simulated (Figure 1). This system is compositionally equivalent to DMS4, barring $\mathrm{Nb}$ and $\mathrm{Hf}$, and henceforth is referred to as model DMS4 system in the rest of this paper. The three visible surfaces in the figure (and all other simulated figures presented in this paper, unless otherwise mentioned) are mutually perpendicular to each other and represent $\{002\}$ planes of the fcc lattice.

Single crystal superalloys such as DMS4 are designed around the two-phase $\gamma+\gamma^{\prime}$ microstructure the origin of which lies in the binary Ni-Al system. Therefore, based on experimental evidence, one expects to see a predominantly two-phase $\gamma+\gamma^{\prime}$ microstructure. However, due to the presence of atoms of many different types, the identification of $\gamma$ and $\gamma^{\prime}$ regions is difficult. As the precise arrangement of atoms in the $\gamma^{\prime}$ phase is known in the case of binary $\mathrm{Ni}_{3} \mathrm{Al}$, the difficulty can be addressed by reducing the multi-component system to a pseudo-binary one comprising Ni-like and Al-like atoms. In order to accomplish this, one needs to know whether an alloying element exhibits Al-like or Ni-like behavior, for example, with respect to partitioning between $\gamma$ and $\gamma^{\prime}$ phases, and/or sub-lattice occupancy in $\gamma^{\prime}$. This necessitates an understanding of ternary Ni-Al-X systems. Therefore we have systematically investigated $\mathrm{Ni}_{3} \mathrm{Al}$ based



Figure 1. Microstructure of simulated model DMS4 system. $\mathrm{Ni}(\mathrm{O}), \mathrm{Al}(\mathrm{O}), \mathrm{Cr}(\mathrm{O}), \mathrm{Co}(\mathrm{O}), \mathrm{Ta}(\mathrm{O}), \mathrm{W}(\mathrm{O})$ and $\operatorname{Re}(\mathrm{O})$. ternary Ni-Al-X $(\mathrm{X}=\mathrm{Cr}, \mathrm{Co}, \mathrm{Ta}, \mathrm{W}$ and $\mathrm{Re})$ systems that can serve as precursors for addressing multi-component systems mimicking the compositions of real nickel base superalloys such as DMS4. For each ternary addition, at least 15 different compositions (Figure 2) were simulated. These compositions have been chosen with $75 \mathrm{Ni}-25 \mathrm{Al}\left(\mathrm{Ni}_{3} \mathrm{Al}\right)$ as reference and incorporation of $3,6,9,12$ or 15 atom percent of the ternary element by substitution in one of three different ways: (a) by replacing $\mathrm{Al}$, (b) by replacing $\mathrm{Ni}$, and (c) by equal replacement of both $\mathrm{Ni}$ and $\mathrm{Al}$. Here, in the interest of brevity, we present our results on $\mathrm{Ni}-\mathrm{Al}-\mathrm{Cr}$ and $\mathrm{Ni}-\mathrm{Al}-\mathrm{Re}$ systems only. These systems have been chosen as they represent the range of complexity observed in our simulations of the different ternary systems. The chromium containing systems exhibit relatively simple microstructures while the rhenium containing systems are considerably complex.

\section{Binary $\mathrm{Ni}-\mathrm{Al}$ system:}

The binary system, consistent with a $\gamma+\gamma^{\prime}$ microstructure, serves as reference for analysis and interpretation of ternary systems.

The simulated system for $75 \mathrm{Ni}-25 \mathrm{Al}$ (corresponding to stoichiometric $\mathrm{Ni}_{3} \mathrm{Al}$ ) is shown in Figure 3a. As expected, the system is composed entirely of different $\mathrm{Ll}_{2}$ domains, corresponding to a $100 \% \gamma^{\prime}$ microstructure. The $\mathrm{L}_{2}$ structure, with stoichiometry $\mathrm{A}_{3} \mathrm{~B}\left(\mathrm{Ni}_{3} \mathrm{Al}\right)$, has a XYXY ... stacking sequence along the $\langle 100\rangle$ directions with alternating planes of pure $\mathrm{A}(\mathrm{Ni})$ and $50 \% \mathrm{~A}(\mathrm{Ni})-50 \% \mathrm{~B}(\mathrm{Al})$. These $\{200\}$ planes can be seen in Figure $3 \mathrm{a}$. The four different $\mathrm{L}_{2}$ variants, indicated by different colors, are shown in Figure $3 \mathrm{~b}$. To obtain this figure, Al atoms that are surrounded by $12 \mathrm{Ni}$ first near neighbors (FNN) have been identified as belonging to $\gamma^{\prime}$. Then, the four variants arise from the specific position occupied by the $\mathrm{Al}$ atom \{one of $(0,0,0),(1 / 2,1 / 2,0),(1 / 2,0,1 / 2)$ or $(0,1 / 2,1 / 2)\}$ with respect to the disordered fcc lattice and an origin defined in the original random configuration. Atoms identified as not belonging to any of the four variants are shown in black, and represent atoms that are either present at anti-phase boundaries (APBs) or at anti-sites within a given domain. The rendering of the structure using variants results in a better visualization aiding the understanding.

The microstructure and the variants in $80 \mathrm{Ni}-20 \mathrm{Al}$ system are given in Figures $3 \mathrm{c}$ and $3 \mathrm{~d}$ respectively. The presence of disordered regions $(\gamma)$ and smaller $\mathrm{L1}_{2}$ domains, relative to the $75 \mathrm{Ni}-25 \mathrm{Al}$ system, can easily be discerned. This demonstrates the feasibility of identifying $\gamma$ and $\gamma^{\prime}$ regions in binary Ni-Al systems

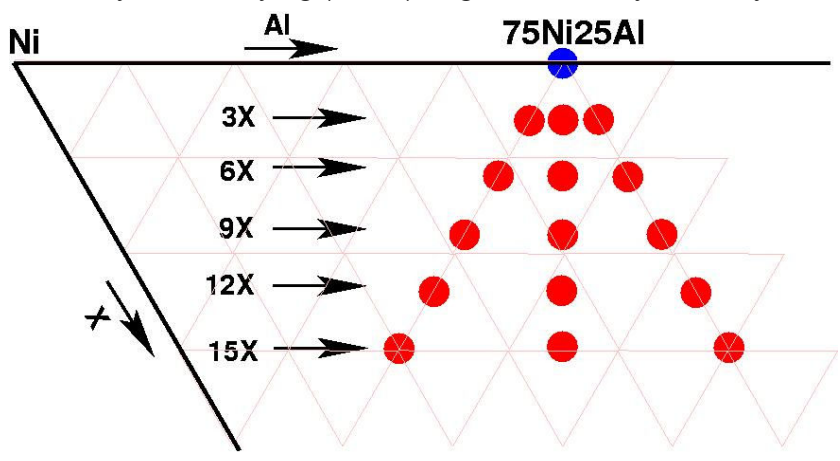

Figure 2. Schematic representation of 15 different ternary compositions selected for MC simulations. 


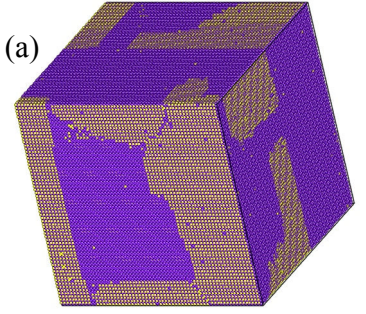

(b)
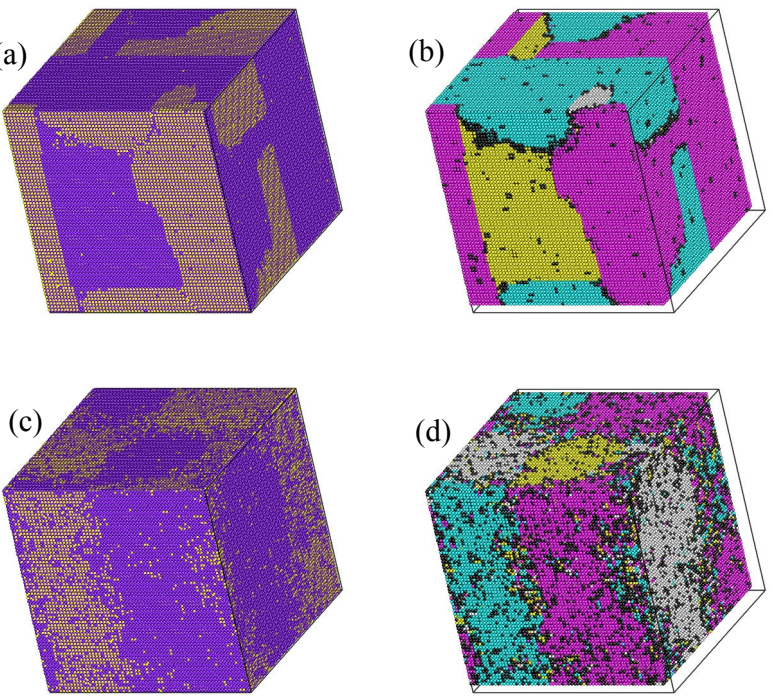

Figure 3. Simulated microstructures of (a) $75 \mathrm{Ni}-25 \mathrm{Al}$ and (c) 80Ni-20Al; $\mathrm{Ni}(\mathrm{O}), \mathrm{Al}(\mathrm{O})$. (b,d) corresponding $\mathrm{L1}_{2}$ variants (cyan, magenta, yellow \& white).

by visual inspection.

\section{$\underline{\text { Ternary } \mathrm{Ni}-\mathrm{Al}-\mathrm{Cr} \text { systems }}$}

The simulated structure of $75 \mathrm{Ni}-22 \mathrm{Al}-3 \mathrm{Cr}$ (Figure $4 \mathrm{a}$ ) shows that $\mathrm{Cr}$ partitions mainly to the disordered regions (which can be seen adjoining $\mathrm{Ll}_{2}$ domains), and increases the number of antisite $\mathrm{Ni}$ atoms. Within the $\mathrm{Ll}_{2}$ regions, $\mathrm{Cr}$ occupies sites of the $\mathrm{Ni}$ sublattice. Rigorous visual examination using AtomEye [7], including sectioning of the system plane by plane at different locations, has revealed that only $\gamma$ and $\gamma^{\prime}$ regions are present in the system.

The observations given above are common to all the $15 \mathrm{Ni}-\mathrm{Al}-\mathrm{Cr}$

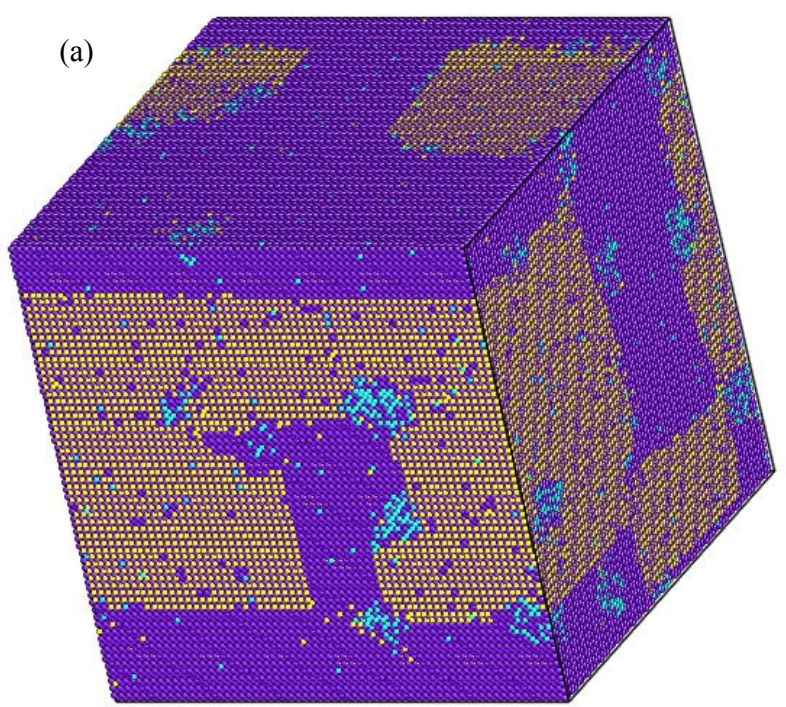

systems investigated. The systems however differ in the relative amounts of $\gamma$ and $\gamma^{\prime}$ phases. In general, increasing the chromium content increases the amount of $\gamma$ phase, as is evident from the microstructure of the $75 \mathrm{Ni}-16 \mathrm{Al}-9 \mathrm{Cr}$ system (Figure $4 \mathrm{~b}$ ). These findings are in broad agreement with those reported in the literature [9].

\section{$\underline{\text { Ternary } \mathrm{Ni}-\mathrm{Al}-\mathrm{Re} \text { systems }}$}

Visual examination of the simulated structure of $75 \mathrm{Ni}-22 \mathrm{Al}-3 \mathrm{Re}$ (Figure 5) reveals that the system is made up only of ordered domains, with the rhenium atoms occupying sites in the $\mathrm{Al}$ sublattice, as a result of which the displaced aluminium atoms are forced to occupy anti-site positions. The situation is similar for the 72Ni-25Al-3Re and the 73.5Ni-23.5Al-3Re systems.

The structures containing 6 atom percent Re are remarkably different. In the aluminium-substituted 75Ni-19Al-6Re system (Figure 6), the rhenium atoms still occupy sites of the $\mathrm{Al}$ sublattice (inferred by visual examination), but the ordering of the sublattices itself is not that of the $\mathrm{L}_{2}$ structure. Rigorous planeby-plane investigation of the structure reveals that the ordering is that of the $\mathrm{DO}_{22}$ structure (Figure 7), which is obtained by a doubling of the $\mathrm{L}_{2}$ unit cell along one of the $<100>$ directions. The tetragonal $\mathrm{DO}_{22}$ structure $\left(\mathrm{A}_{3} \mathrm{~B}\right.$ stoichiometry) has a XYZYXYZY ... stacking (Figure 7a) along the unique direction, with alternating planes of pure $\mathrm{A}$ and $50 \% \mathrm{~A}-50 \% \mathrm{~B}$. The $\mathrm{X}$ and $\mathrm{Z}$ layers are related to each other by a $1 / 2<110>$ in-plane translation. The change of symmetry from cubic to tetragonal gives rise to three $\mathrm{DO}_{22}$ variants for every $\mathrm{Ll}_{2}$ variant, resulting in a total of $12 \mathrm{DO}_{22}$ variants (as there are $4 \mathrm{Ll}_{2}$ variants). In the simulated structures, the interfaces between the different variants, as in the case of $\mathrm{L1}_{2}$, are characterized by APBs. Figure $7 \mathrm{~b}$ shows the arrangement of atoms in a plane perpendicular to either [100] or [010], for the tetragonal axis along [001]. This arrangement of atoms is clearly evident in the simulated microstructure shown in Figure 6. Detailed scrutiny of this microstructure confirms that $\mathrm{DO}_{22}$ is the majority phase in this system. Amongst the other two systems with 6 atom percent Re, the equally substituted system



Figure 4. Simulated microstructures of (a) 75Ni-22Al-3Cr and (b) 75Ni-16Al-9Cr systems. $\mathrm{Ni} \mathrm{(O} \mathrm{),} \mathrm{Al}(\mathrm{O})$ and $\mathrm{Cr}(\mathrm{O})$. 


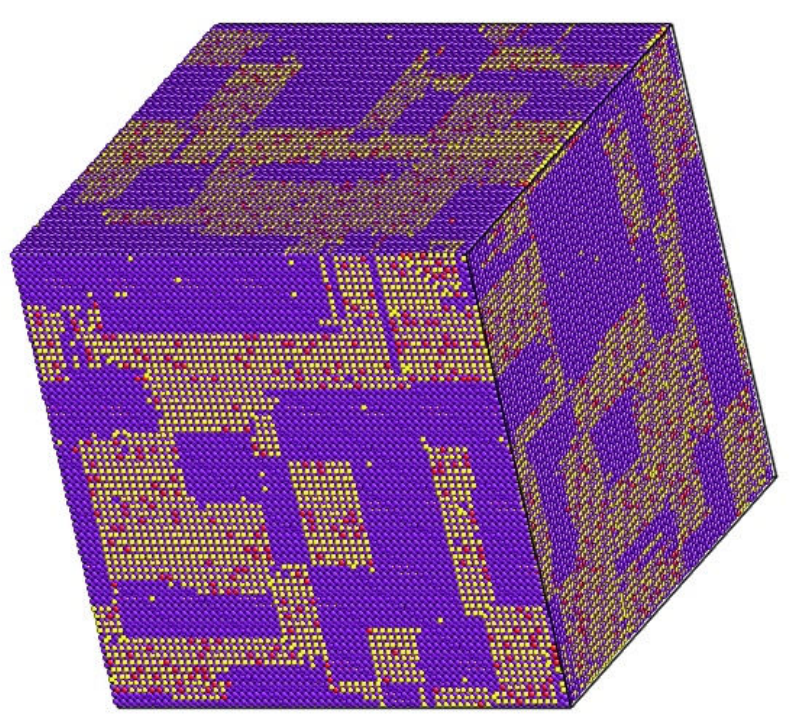

Figure 5. Simulated microstructure of $75 \mathrm{Ni}-22 \mathrm{Al}-3 \mathrm{Re}$.

$\mathrm{Ni}(\mathrm{O}), \mathrm{Al}(\mathrm{O})$ and $\operatorname{Re}(\mathrm{O})$.

has microstructure similar to that of the aluminium-substituted system. In contrast, $\gamma$ ' continues to be the dominant phase in the nickel-substituted system (69Ni-25Al-6Re).

The aluminium-substituted systems with higher amounts of Re are qualitatively similar to the $75 \mathrm{Ni}-19 \mathrm{Al}-6 \mathrm{Re}$ system, with the $75 \mathrm{Ni}-$ 10Al-15Re system being composed almost entirely of the $\mathrm{DO}_{22}$ phase. In these systems, Re atoms are found to occupy sites of the Al sublattice.

In contrast, the microstructure of nickel-substituted $66 \mathrm{Ni}-25 \mathrm{Al}-$ 9Re system (Figure 8) is quite complex. $\gamma^{\prime}$ regions can still be discerned, but the presence of another ordered structure, not $\mathrm{DO}_{22}$,

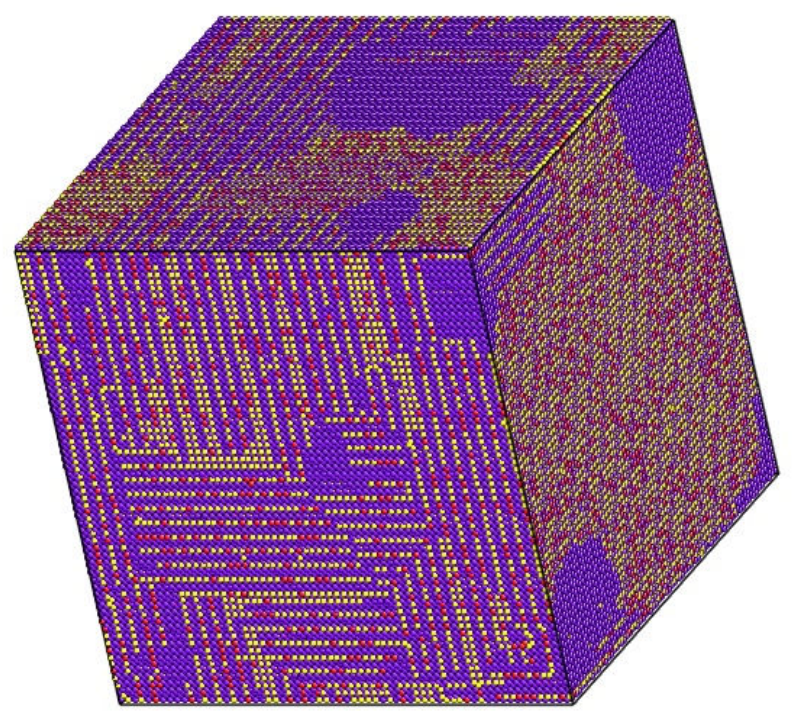

Figure 6. Simulated microstructure of 75Ni-19Al-6Re. $\mathrm{Ni}(\mathrm{O}), \mathrm{Al}$ ( O ) and $\operatorname{Re}(\mathrm{O})$.



(b)

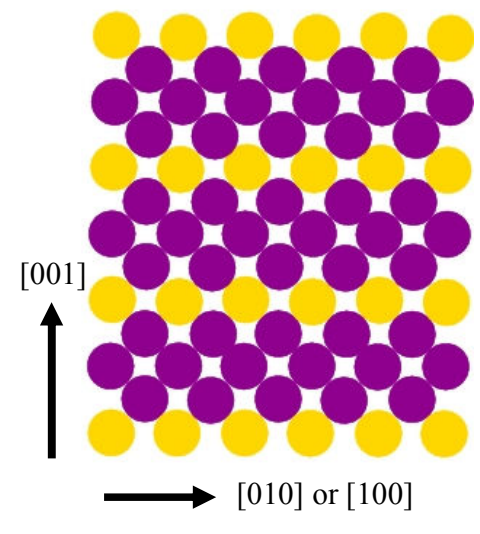

Figure 7. (a) $\mathrm{DO}_{22}$ structure with $\mathrm{A}_{3} \mathrm{~B}$ stoichiometry (b) arrangement of atoms on a (200) or (020) plane

can also be seen. This structure seems to be devoid of $\mathrm{Al}$ and is made up exclusively of $\mathrm{Ni}$ and $\mathrm{Re}$. Closer examination (plane-byplane) of this structure shows the presence of a unit cell made up of two cubic unit cells, but with arrangement of atoms (Figure 9) different from $\mathrm{DO}_{22}$. This ordered structure has $\mathrm{AB}$ stoichiometry, belongs to the tetragonal $\mathrm{I}-4 \mathrm{~m} 2$ space group and is designated "40" in the Strukturbericht notation [10]. This structure has a XYZWXYZW... stacking sequence along the unique direction, with all planes having a $50 \% \mathrm{~A}-50 \% \mathrm{~B}$ stoichiometry. The ' $\mathrm{X}$ ' and ' $\mathrm{Z}$ ' planes are related as in the $\mathrm{DO}_{22}$ structure, and the ' $\mathrm{W}$ ' and ' $\mathrm{Y}$ ' planes are related by an identical translation as ' $\mathrm{X}$ ' and ' $Z$ '. This stacking gives rise to the characteristic 'zipper' structure, in the (200) and the (020) planes (Figure 9b), of two successive rows of A atoms followed by two successive rows of B atoms.

It can be seen that the complexity characteristic of a multicomponent system such as DMS4 (Figure 1) is already



Figure 8. Simulated microstructure of 66Ni-25Al9Re. Some "40" regions are encircled. Ni ( ) , Al ( O ) and $\operatorname{Re}(\mathrm{O})$. 

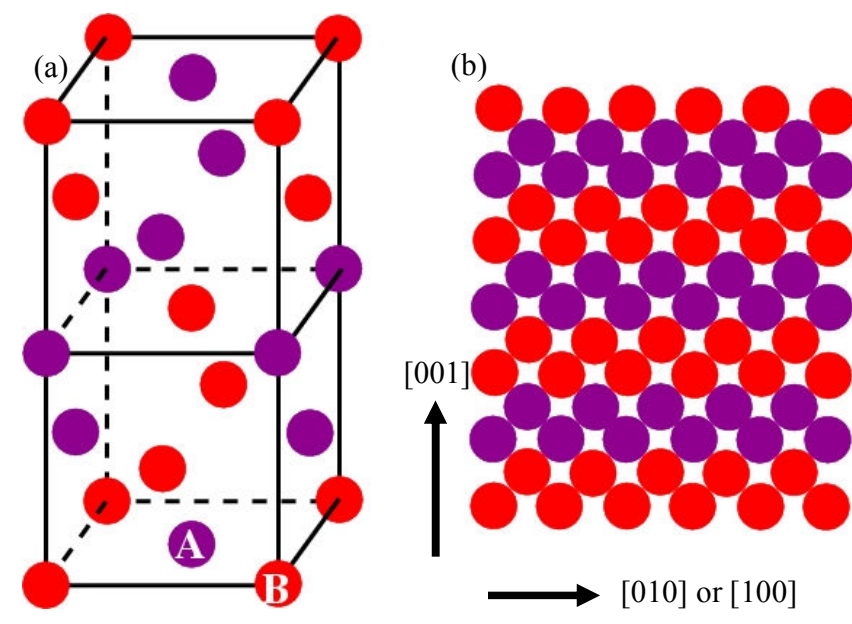

Figure 9. (a) Unit cell of " 40 " structure (b) arrangement of atoms on a (200) or (020) plane

evident in this ternary system - several different phases are present, and visual examination of the simulated microstructures is insufficient to describe the attributes of the microstructure, for example, the constituent phases, their relative volume fractions and their composition. A more robust approach is essential to address these issues.

\section{$\underline{\text { Phase Identification and Quantification }}$}

The visual examination of the different systems presented above reveals, depending on the system composition, the presence of up to three ordered phases ( $\mathrm{L}_{2}, \mathrm{DO}_{22}$ and " 40 ") and the disordered fcc phase $(\gamma)$ in the simulated microstructures. It is important to note that the first near neighbor (FNN) coordination shell is identical in both $\mathrm{L}_{2}$ and $\mathrm{DO}_{22}$ structures. This implies that, if one uses only FNN shell for phase identification, the $\mathrm{DO}_{22}$ structure that is present would be identified, erroneously, as $\mathrm{L1}_{2}$. The second near neighbor (SNN) coordination needs to be taken into account for correct phase identification. We have therefore developed a code to identify the phases based on the environment of a selected atom by considering FNN and SNN information (Table I). The algorithm, on which this code is based, is presented in Appendix in the form of pseudocode.

Table I: First and second near neighbor coordination in $\mathrm{L}_{2}$, $\mathrm{DO}_{22}$ and " 40 " structures

\begin{tabular}{|c|c|c|c|c|}
\hline Center atom & \multicolumn{2}{|c|}{ FNN } & \multicolumn{2}{c|}{ SNN } \\
\hline $\left.\mathbf{L 1}_{\mathbf{2}} \mathbf{( A}_{\mathbf{3}} \mathbf{B}\right)$ & A & B & A & B \\
\hline A & 8 & 4 & 6 & 0 \\
\hline B & 12 & 0 & 0 & 6 \\
\hline DO $\left._{\mathbf{2 2}} \mathbf{( A}_{\mathbf{6}} \mathbf{B}_{\mathbf{2}}\right)$ & A & B & A & B \\
\hline $\mathbf{A ~ ( A 1 ) ~}$ & 8 & 4 & 4 & 2 \\
\hline $\mathbf{A ~ ( A 2 ) ~}$ & 8 & 4 & 6 & 0 \\
\hline B & 12 & 0 & 2 & 4 \\
\hline${\left.\text { 40" (A4 } \mathbf{B}_{4}\right)}^{\mathbf{4}}$ & A & B & A & B \\
\hline A & 4 & 8 & 4 & 2 \\
\hline B & 8 & 4 & 2 & 4 \\
\hline
\end{tabular}

The code provides for the presence of only two types of atoms A and $\mathrm{B}$ (or $\mathrm{Ni}$ and $\mathrm{Al}$ ); ternary and higher order systems are reduced to pseudo-binary systems using visual examination to determine whether the other atoms exhibit either Ni- or Al- like behavior. The results of applying the code to the various systems are presented below.

\section{Binary $\mathrm{Ni}-\mathrm{Al}$ systems:}

The $75 \mathrm{Ni}-25 \mathrm{Al}$ system is found to have $89 \% \gamma^{\prime}$ phase, $\sim 10 \%$ $\mathrm{DO}_{22}$ phase and less than $1 \%$ of $\gamma$ and unresolved phases. Visual examination had shown that the system (Figure 3a) is composed entirely of $\gamma^{\prime}$ phase. When the distribution of phases is rendered (Figure 10a), instead of the actual atoms (as in Figure 3a), it can be seen that the regions identified by the code as $\mathrm{DO}_{22}$ are predominantly APBs between various $\mathrm{L}_{2}$ domains. This is an outcome of the inherent nature of the algorithm used. In addition, a few antisite regions have also been identified as $\mathrm{DO}_{22}$. The phase distribution for the $80 \mathrm{Ni}-20 \mathrm{Al}$ system is shown in Figure 10b. In this system, the calculated amount of phases (approx.) is as follows: $23 \% \gamma, 30 \% \gamma^{\prime}, 35 \% \mathrm{DO}_{22}$ and $12 \%$ unresolved phase. Truly, this might be interpreted as a system with approximately $23 \% \gamma$ and $77 \% \gamma$ '. The apparently large amounts of $\mathrm{DO}_{22}$ as well as that of the unresolved phase is due to the increased area fraction of both APBs as well as $\gamma / \gamma^{\prime}$ interfaces in this system relative to the $75 \mathrm{Ni}-25 \mathrm{Al}$ system. The interfaces are regions wherein the bulk coordination of the atoms is disrupted thereby leading to difficulties in the correct phase identification. As the amount of the disordered phase increases, it becomes difficult to distinguish between extremely small (comprising 2-3 cubic unit cells) regions of the disordered phase and the disruption in order at interphase interfaces. Thus, it is obvious that, the quantification still needs to be supplemented by visual examination of the systems.

\section{Ternary $\mathrm{Ni}-\mathrm{Al}-\mathrm{Cr}$ systems}

Based on visual inspection, $\mathrm{Cr}$ has been treated to be Ni-like for reduction of the systems to corresponding pseudo-binaries. The phase distribution in $75 \mathrm{Ni}-22 \mathrm{Al}-3 \mathrm{Cr}$ and $75 \mathrm{Ni}-16 \mathrm{Al}-9 \mathrm{Cr}$ systems is shown in Figures $11 \mathrm{a}$ and $11 \mathrm{~b}$ respectively. These can be compared with the microstructure given in Figures $4 \mathrm{a}$ and $4 \mathrm{~b}$. The increase in the amount of $\gamma$ phase in the $9 \mathrm{Cr}$ system can clearly be seen, demonstrating the benefit of using the phase identification for analyzing the simulated microstructures.
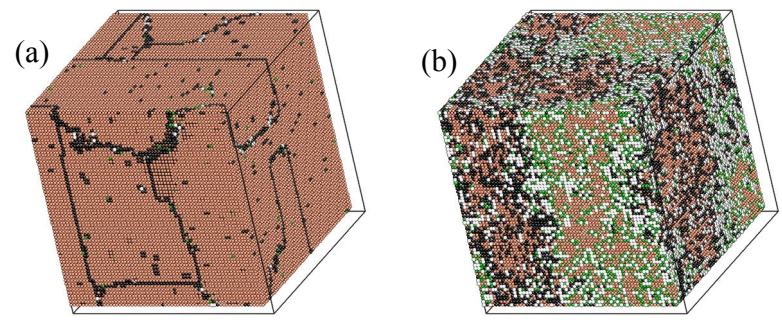

Figure 10. Phase distribution in (a) $75 \mathrm{Ni}-25 \mathrm{Al}$ (b) $80 \mathrm{Ni}$ 20Al. $\gamma$ (white), $\gamma^{\prime}$ (orange), $\mathrm{DO}_{22}$ (black) and unresolved (green) 

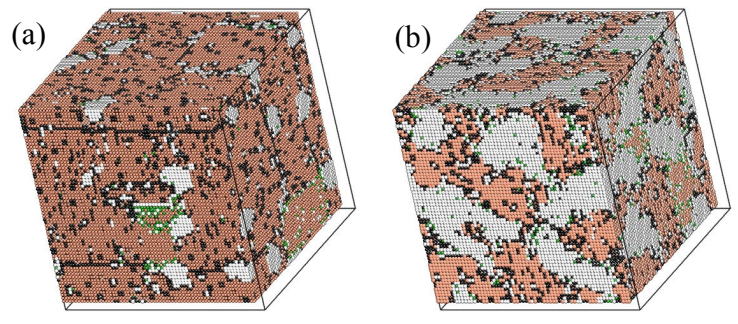

Figure 11. Phase distribution in (a) $75 \mathrm{Ni}-22 \mathrm{Al}-3 \mathrm{Cr}$ (b) $75 \mathrm{Ni}-16 \mathrm{Al}-9 \mathrm{Cr}$. $\gamma$ (white), $\gamma^{\prime}$ (orange), $\mathrm{DO}_{22}$ (black) and unresolved (green).

The relative volume fractions of the different phases in the Ni-Al$\mathrm{Cr}$ ternary systems investigated are shown in Figure 12. It can be seen that the volume fraction of $\gamma$ (Figure 12a) increases with increase in $\mathrm{Cr}$ content. As it is known through visual analysis that the Ni-Al-Cr ternary systems investigated possess a two phase $\gamma+$ $\gamma^{\prime}$ microstructure, the volume fraction of $\mathrm{DO}_{22}$ (Figure 12c) arises from $\mathrm{L}_{2}$ APBs. Accordingly, the actual $\mathrm{L1}_{2}$ volume fraction is obtained from adding the identified $\mathrm{Ll}_{2}$ and $\mathrm{DO}_{22}$ fractions (Figure 12d). The decrease in volume fraction of $\gamma^{\prime}$, with increasing $\mathrm{Cr}$, is strongest in the case of Al-substituted systems. This is due to the fact that chromium exhibits a tendency to partition strongly to the $\gamma$ phase which implies that the extent of $\gamma^{\prime}$ is largely governed by the availability of $\mathrm{Al}$ in the system. This is consistent with the reported $\mathrm{Ni}$-Al-Cr ternary phase diagram [9].
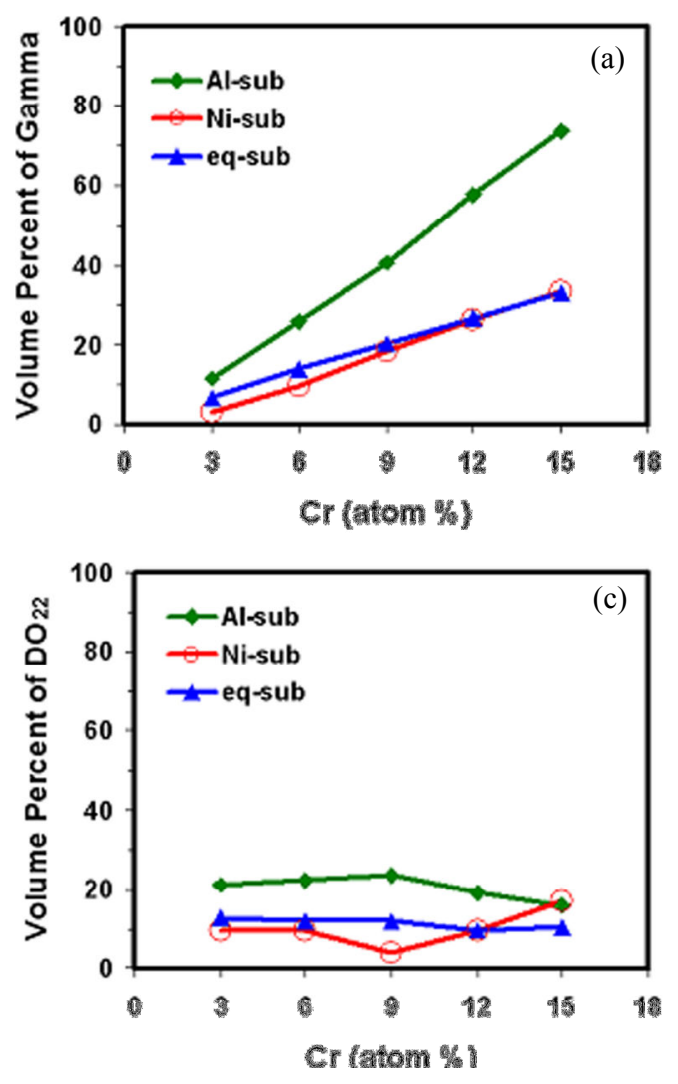

Figure 12. Volume fractions of the different phases observed in Ni-Al-Cr ternary systems. (a) $\gamma$ (b) $\mathrm{L1}_{2}$ (c) $\mathrm{DO}_{22}$ and (d) $\mathrm{Ll}_{2}+\mathrm{DO}_{22}$. (Figure 13b) can be seen clearly. Re ternary systems investigated are shown in Figure 14. remains insensitive to $R e$ content. concentration $\geq 9$ atom percent. marginal decrease in the fraction of $\mathrm{DO}_{22}$.
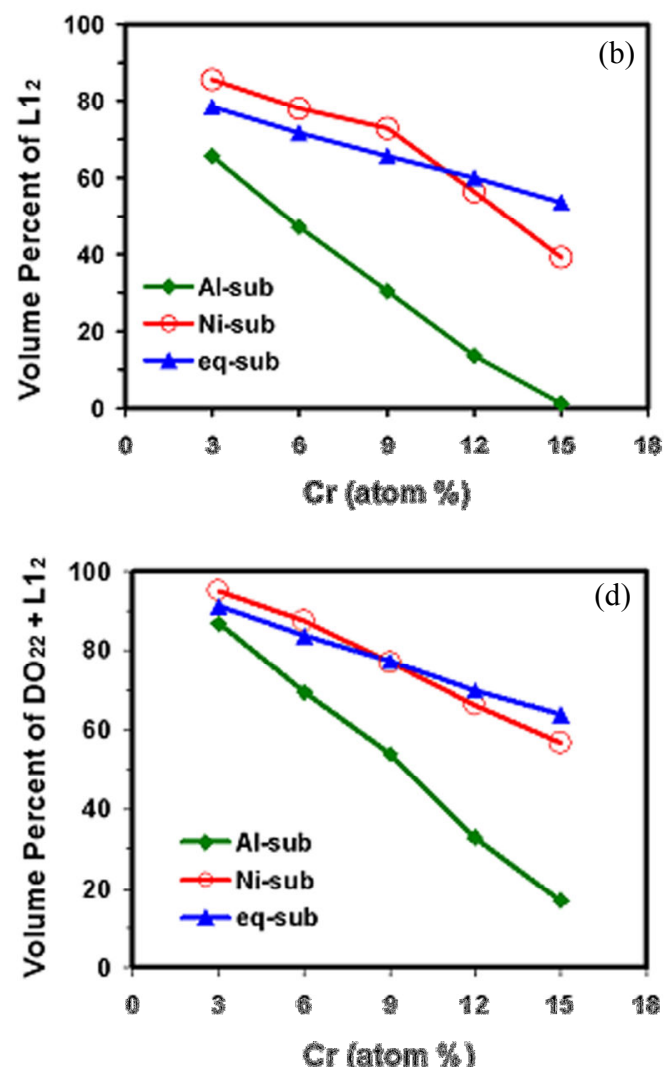

$\underline{\text { Ternary } \mathrm{Ni}-\mathrm{Al}-\mathrm{Re} \text { systems }}$

Based on visual inspection, Re has been treated to be Al-like for reduction of the systems to corresponding pseudo-binaries. The phase distribution in $75 \mathrm{Ni}-22 \mathrm{Al}-3 \mathrm{Re}$ and $66 \mathrm{Ni}-25 \mathrm{Al}-9 \mathrm{Re}$ systems is shown in Figures $13 \mathrm{a}$ and $13 \mathrm{~b}$ respectively. These can be compared with the microstructure given in Figures 5a and 8. The 3Re system is confirmed to be made up primarily of $\mathrm{L}_{2}$ regions. The presence of $\mathrm{Ll}_{2}, \mathrm{DO}_{22}$ and "40" regions in the $9 \mathrm{Re}$ system

The relative volume fractions of the different phases in the Ni-Al-

Ni-substituted systems: The fraction of " 40 " and $\gamma$ increases with increasing $\mathrm{Re}$ while that of $\mathrm{Ll}_{2}$ decreases. The fraction of $\mathrm{DO}_{22}$

Al-substituted systems: The amount of $\gamma$ phase is insensitive to Re content while the amount of " 40 " is negligible. With increasing $\mathrm{Re}$, the fraction of $\mathrm{DO}_{22}$ increases at the expense of $\mathrm{Ll}_{2}$; the system is nearly $(>90 \%)$ single phase $\mathrm{DO}_{22}$ for $\mathrm{Re}$

Equally substituted systems: The amount of $\gamma$ phase increases marginally with increasing $\mathrm{Re}$ and saturates at about 9 atom percent Re. While there is no "40" phase in the system with 3 atom percent Re, it starts appearing at 6 at. \% Re and increases with further increase in Re content. The fraction of $\mathrm{Ll}_{2}$ phase decreases up to 9 at. \% Re addition where it reaches a minimum. Correspondingly the $\mathrm{DO}_{22}$ fraction increases and reaches a maximum for this composition. Further addition of Re leads to a 

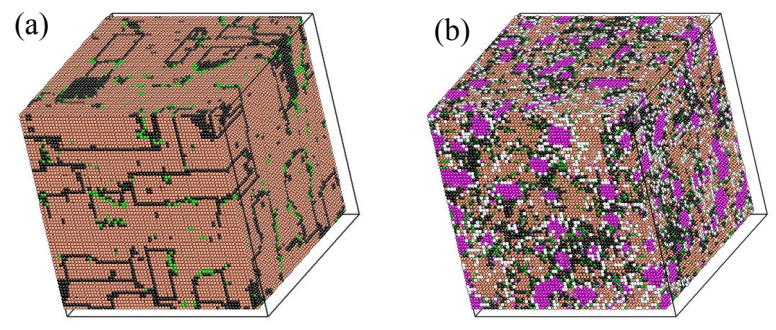

Figure 13. Phase distribution in (a) $75 \mathrm{Ni}-22 \mathrm{Al}-3 \mathrm{Re}$ (b) 66Ni-25Al-9Re. $\gamma$ (white), $\gamma^{\prime}$ (orange), $\mathrm{DO}_{22}$ (black), "40" (magenta) and unresolved (green).

Experimental evidence for the partitioning of Re to $\gamma^{\prime}$ in ternary alloys has been reported in the literature. Using energy dispersive spectroscopy in TEM, Volek et al [11] have reported partition coefficient $\left(\mathrm{k}=\mathrm{c}^{\gamma^{\gamma}} / \mathrm{c}^{\gamma}\right)$ of $1.85 \pm 0.30$ for a model ternary alloy (82.9Ni-16.2Al-0.9Re, in at. \%). Our calculation on system with identical composition yielded a partition coefficient of 2.01. Further, first principles calculations of a $\gamma / \gamma^{\prime}$ interface [12], performed for different positions of Re in the $\gamma^{\prime}$ lattice, predict lower energy for Re occupying $\mathrm{Al}$ sublattice site rather than $\mathrm{Ni}$ sublattice site. Other MC simulations [13] on model Ni-base superalloys show the nearest neighbor Re-Re and Re-Ni coordination to be similar to those of $\mathrm{Al}-\mathrm{Al}$ and $\mathrm{Al}-\mathrm{Ni}$, respectively. This confirms that Re has Al-like behavior in ordered structures. Thus, in agreement with our results, there seems to be strong evidence in the literature for the partitioning of $\operatorname{Re}$ to $\gamma^{\prime}$ in ternary systems, and for its tendency to replace Al in $\gamma^{\prime}$, especially for systems with $\operatorname{Re}$ content less than $\sim 3$ atom percent. At higher Re concentrations, the presence of $\mathrm{DO}_{22}$ and " 40 " phases in the microstructure may be due to the tendency of Re to form the wellknown topologically close packed (TCP) phases. It must be noted that any ordered structure observed must necessarily conform to the basic fcc lattice which has been used in our simulation. Thus, observed structures such as $\mathrm{DO}_{22}$ and "40" may therefore simply be manifestations of other ordered structures, notably those based on hexagonal lattices $[14,15]$ which are prevalent in nickel base superalloys. The presence of $\mathrm{DO}_{22}$ and "40" structures with stoichiometry of $\mathrm{W}(\mathrm{Ni}, \mathrm{Cr})_{3}$ and $\mathrm{W}(\mathrm{Ni}, \mathrm{Cr})$ respectively, have been reported in $\mathrm{MC}$ simulations on tungsten containing nickel base quaternary alloys [16]. These simulations were also based on the fcc lattice.

\section{Model DMS4 system}

The results from the simulation of ternary systems, at specific ternary element concentrations of interest to model DMS4 system, revealed that $\mathrm{Cr}$ and $\mathrm{Co}$ exhibited Ni-like behavior while Ta, $\mathrm{W}$


\begin{tabular}{l|l} 
(a) & (b) \\
\hline (c) & (d)
\end{tabular} 18

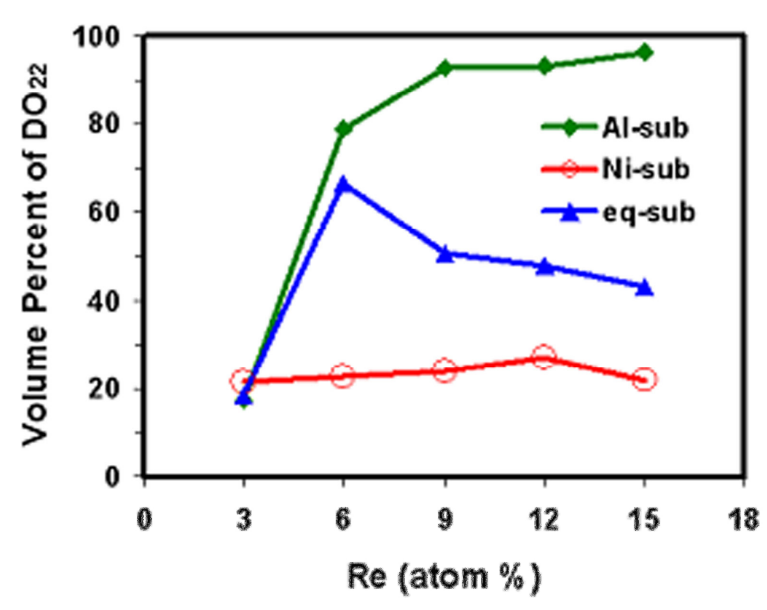

Figure 14. Volume fractions of different phases in ternary Ni-Al-Re systems: (a) $\gamma$. Inset shows an enlarged view (b) $\mathrm{L1}_{2}$ (c) $\mathrm{DO}_{22}$ and (d) " 40 ". 


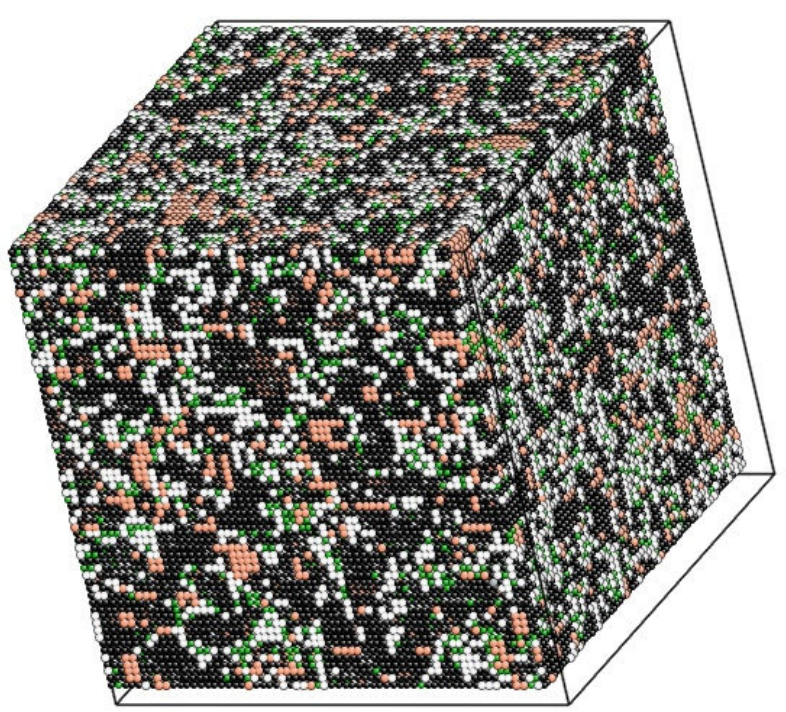

Figure 15. Phase distribution in DMS4 alloy. $\gamma$ (white), $\gamma$ ' (orange), $\mathrm{DO}_{22}$ (black) and unresolved (green).

and Re exhibited Al-like behavior. This information has been used to reduce the multi-component DMS4 system to a pseudo-binary one for phase identification and quantification. The system contains approximately $23 \% \gamma, 58 \% \mathrm{DO}_{22}$ and only $8 \% \gamma \gamma$. The remaining $11 \%$ is unresolved and represents small ( $\sim 1-2$ unit cells) disordered regions or interphase interfaces that are not APBs. Based on results from our experiments on DMS4, as well as those reported in the literature on similar alloys, the dominating presence of the $\mathrm{DO}_{22}$ phase (Figure 15) is unexpected. This may be due to the fact that the pairwise interatomic potentials that have been used are too simplistic and do not accurately describe the interatomic interactions in the multi-component alloy.

Several reports in the literature [5, 16-19] have used identical or similar potentials to investigate the microstructure of multicomponent superalloys, and report the presence of only $\gamma$ and $\gamma^{\prime}$ phases. Saito and Harada [5] have used identical pairwise potentials to simulate the microstructure of TMS63 and CMSX4 and have reported the partitioning of alloying elements between $\gamma$ and $\gamma^{\prime}$. They do not report the presence of any $\mathrm{DO}_{22}$ phase. However, our simulations on identical systems yield microstructures with considerable amount of $\mathrm{DO}_{22}$ phase, which is similar to our results from DMS4. As we have mentioned earlier, $\mathrm{L}_{2}$ and $\mathrm{DO}_{22}$ cannot be distinguished based on FNNs alone. It is possible that Saito and Harada [5] have only used FNN environments for phase identification.

In order to compare our results with literature reports, we repeated our phase identification considering only FNN environment on systems that were simulated using up to $3 \mathrm{NN}$ interactions. In this case, we found the model DMS4 system to contain $77 \% \gamma^{\prime}$ and $23 \% \gamma$ (Figure 16). Likewise, the simulated TMS63 and CMSX4 systems were found to contain $79 \%$ and $74 \% \gamma^{\prime}$ respectively. Our result for CMSX4 is in reasonable agreement with APFIM results reported in the literature $[19,20]$.

These observations suggest that, while the interatomic potentials used favor $\mathrm{DO}_{22}$ over $\mathrm{L}_{2}$ in the case of multi-component alloys,



Figure 16. Phase distribution in DMS4 alloy considering only first near neighbors for phase identification. $\gamma$ (white) and $\gamma^{\prime}$ (orange)

ignoring the difference between the two structures does seem to result in volume fractions of $\gamma^{\prime}$ that are reasonable. However, the implications of this approach remain to be understood.

Extending the same approach, the composition of $\gamma$ and $\gamma^{\prime}$, and the partitioning of different alloying elements, considering only up to FNN for phase identification, can be determined (Table II). It can be seen that $\mathrm{Cr}$ and Co partition to $\gamma$ while $\mathrm{Al}$, Ta and Re partition to $\gamma^{\prime}$. Ni and $\mathrm{W}$ show only weak partitioning. This can be compared with the partitioning of alloying elements determined from atom probe experiments (Figure 17) on the fully aged DMS4 alloy (Table II). While Re, Cr and Co show partitioning into $\gamma, \mathrm{Al}$ and $\mathrm{Ta}$ exhibit partitioning into $\gamma^{\prime}$. Ni (not shown), $\mathrm{Nb}$ and $\mathrm{W}$ exhibit relatively weak partitioning. Qualitatively, both MCS and atom probe experiments are in agreement on the partitioning of all



Figure 17. 3D atom maps from a fully aged DMS4 sample. Ni map is not shown. 
Table II: Composition of $\gamma$ and $\gamma^{\prime}$, and partition coefficients in DMS4, obtained from atom probe and Monte Carlo simulations.

\begin{tabular}{|c|r|r|r|r|r|r|}
\hline \multirow{2}{*}{ Element } & \multicolumn{3}{|c|}{ 3D Atom Probe } & \multicolumn{3}{c|}{ MC simulations } \\
\cline { 2 - 7 } & $\begin{array}{c}\mathrm{c}^{\gamma}, \\
\text { at. \% }\end{array}$ & $\begin{array}{c}\mathrm{c}^{\gamma}, \\
\text { at. \% }\end{array}$ & $\mathrm{k}^{\#}$ & $\begin{array}{c}\mathrm{c}^{\gamma^{\prime}}, \\
\text { at. \% }\end{array}$ & $\begin{array}{c}\mathrm{c}^{\gamma}, \\
\text { at. \% }\end{array}$ & $\mathrm{k}^{\#}$ \\
\hline $\mathrm{Ta}$ & 5.42 & 0.64 & 8.47 & 3.71 & 1.74 & 2.13 \\
\hline $\mathrm{Al}$ & 16.63 & 2.47 & 6.73 & 13.99 & 6.92 & 2.04 \\
\hline $\mathrm{Ni}$ & 70.81 & 67.75 & 1.05 & 71.08 & 78.74 & 0.90 \\
\hline $\mathrm{Nb}$ & 2.02 & 1.93 & 1.05 & \multicolumn{3}{c|}{ Not considered } \\
\hline $\mathrm{W}$ & 2.39 & 3.03 & 0.79 & 2.07 & 1.36 & 1.52 \\
\hline $\mathrm{Co}$ & 1.31 & 6.89 & 0.19 & 3.92 & 5.62 & 0.70 \\
\hline $\mathrm{Cr}$ & 0.97 & 8.40 & 0.12 & 2.37 & 4.79 & 0.49 \\
\hline $\mathrm{Re}$ & 0.29 & 8.79 & 0.04 & 2.85 & 0.82 & 3.48 \\
\hline
\end{tabular}

${ }^{\#} \mathrm{k}=\mathrm{c}^{\gamma^{\gamma}} / \mathrm{c}^{\gamma}=$ partition coefficient

elements other than Re and W. The extent of partitioning though is considerably different, with the experiments showing much stronger partitioning. Volek et al. [11] have reported that Re, Mo and $\mathrm{W}$ exhibit a reversal of partitioning between simpler ternary (or quaternary) systems and multicomponent superalloys. These elements partition preferentially to $\gamma^{\prime}$ in the former and to $\gamma$ in the latter, with the effect being most pronounced in the case of Re. While the reasons underlying this behavior have not been elaborated, it is perhaps due to complex multi-species interactions that are likely to prevail in multicomponent systems. This complexity has not been captured in the simplistic L-J type pairpotentials used in the current simulations which therefore do not predict this reversal of partitioning of Re and $\mathrm{W}$, on transitioning from model ternary or quaternary systems to realistic multicomponent superalloy systems. Nevertheless, as the partitioning of $\mathrm{W}$ is in any case weak, further discussion is focused on the partitioning of Re in multicomponent superalloys.

Several studies show $\operatorname{Re}$ partitions strongly to $\gamma$ in multicomponent alloys [19-24]. Further, Babu et al [24] report strong enhancement of Re concentration in $\gamma$, at the $\gamma / \gamma$ 'interface. They show the accumulation of Re in the form of a 'bow-wave' ahead of the growing $\gamma^{\prime}$ particles. Subsequent homogenization within $\gamma$ is diffusion limited and therefore the interface enhancement of $\mathrm{Re}$ does not usually disappear. The local concentration of $\operatorname{Re}$ at the $\gamma / \gamma^{\prime}$ interface has been estimated to be as high as 15 at. $\%$. Our atom probe results on fully aged DMS4 show that the Re concentration at the $\gamma / \gamma^{\prime}$ interface often exceeds 25 atom percent [25].

\section{Summary and Conclusions}

Monte Carlo simulations of ternary and multi-component Ni-base superalloys have been performed and APFIM has been used to characterize fully aged DMS4 superalloy. While experimental systems are composed essentially of two phases ( $\gamma$ and $\left.\gamma^{\prime}\right)$, the simulated systems showed the existence of other phases. An algorithm has been devised to identify and quantify $\gamma, \gamma$, $\mathrm{DO}_{22}$ and " 40 " phases in simulated systems and applied successfully to model Ni-Al-Cr, Ni-Al-Re and multicomponent systems. Results from simulations show the well-known behavior of $\mathrm{Cr}$ as $\gamma$ promoter. In ternary $\mathrm{Ni}-\mathrm{Al}-\mathrm{Re}$ systems, Re has been found to partition to $\gamma^{\prime}$ phase which is in agreement with experimental findings [11].
Simulation of multicomponent alloys with reported $\gamma+\gamma$ ' microstructures show a preponderance of $\mathrm{DO}_{22}$ phase instead of $\mathrm{L1}_{2}\left(\gamma^{\prime}\right)$. This indicates that the current description of the interatomic interactions, that are pair-wise, is inadequate for describing/predicting the phases and alloying element partitioning in multicomponent systems. An assessment of the literature indicates that better interatomic potentials such as embedded atom potentials, are available, only for few systems where there are three or more components. Clearly, there is an urgent need for the development of such potentials if Monte Carlo (and similar atomistic) simulations are to be used effectively in the design and development of newer nickel base superalloys as well as to gain deeper understanding of physical metallurgy of existing superalloys.

\section{Acknowledgements}

This research was funded by Defence Research and Development Organization (DRDO), India, under Project DMR-254, 'Science and Design of Materials at Atomic Scale'. The permission of Director, Defence Metallurgical Research Laboratory (DMRL), Hyderabad, to publish the results is gratefully acknowledged. The authors are grateful to Dr. D. Banerjee, Chief Controller, DRDO for his keen interest, constant encouragement and guidance throughout the project.

\section{References}

1. N. Das, S. Singh, N. Hazari, D. Chaterjee and V.V.N.S.S.C. Praveen, "Indigenous Cast Superalloys and Investment Casting Technology for Gas Turbine Components," Metals, Materials and Processes 19 (2007) 189-202.

2. N. Das, US Patent 5916384, 1999.

3. R. Sankarasubramanian and R. Balamuralikrishnan, "Monte Carlo Simulations for Microstructural Evolution - Part I: Code Development" (Report DMRL TR 2004 360, Defence Metallurgical Research Laboratory, 2004).

4. M. Enomoto and H. Harada, "Analysis of $\gamma^{\prime} / \gamma$ Equilibrium in Ni-Al-X Alloys by the Cluster Variation Method with the Lennard-Jones Potential," Metall. Trans. A, 20 (1989) 649-664.

5. Y. Saito and H. Harada, "The Monte Carlo Simulation of Ordering Kinetics in Ni-base Superalloys," Mater. Sci. Eng. A, 223 (1997), 1-9.

6. K. Kawasaki, "Diffusion Constants Near the Critical Point for Time-dependent Ising Models - I," Phys. Rev., 145 (1966), 224-230.

7. J. Li, "AtomEye: An Efficient Atomistic Configuration Viewer," Modelling Simul. Mater. Sci. Eng., 11 (2003) 173-177.

8. M.K. Miller and G.D.W. Smith, Atom Probe Microanalysis: Principles and Applications to Materials Problems, (Pittsburgh, PA: Materials Research Society, 1989), 43-50.

9. S. Ochiai, Y. Oya, and T. Suzuki, "Alloying Behaviour of $\mathrm{Ni}_{3} \mathrm{Al}$," Acta Metallurgica, 32 (1984) 289-298. 
10. World wide web: http://cst-www.nrl.navy.mil/lattice/struk/NbP.html

11. A. Volek, F. Pyczak, R.F. Singer, and H. Mughrabi, "Partitioning of Re between $\gamma$ and $\gamma$ ' Phase in Nickelbase Superalloys," Scripta Mater., 52 (2005) 141-145.

12. K. Chen and L.R. Zhao, "Atomic Mechanism of the Re and $\mathrm{Ru}$ Strengthening Effect on the $\gamma / \gamma$ ' Interface of Nibased Single-Crystal Superalloys: A First-Principles Study," Phil. Mag. 83 (2003) 1685-1698.

13. J. Buršík, "Quantitative Analysis of Atomic Configurations of Two-phase Ni-based Alloys Generated by Monte Carlo Simulation," J. Alloys and Comp., 378 (2004) 66-70.

14. C.M.F. Rae and R.C. Reed, "The Precipitation of Topologically Close-Packed Phases in Rheniumcontaining Superalloys," Acta Mater. 49 (2001) 41134125 .

15. Madeline Durand-Charre, The Microstructure of Superalloys (Amsterdam: Gordon and Breach Science Publishers, 1997), 48.

16. J. Buršík, "Phase Constitution of Ni-based Quaternary Alloys studied by Monte Carlo Simulation," Computer Physics Communications, 147 (2002) 162-165.

17. Y. Saito, "The Monte Carlo Simulation of the Ordering Kinetics of fcc to $\mathrm{L}_{2}$ Structure in Ni-Al-X Ternary Alloys," Mater. Sci. Eng. A, 223 (1997) 10-16.

18. H. Harada and H. Murakami, "Design of Ni-Base Superalloys," ed. T. Saito, Springer Series in Materials Science, vol. 34, (Springer-Verlag Berlin Heidelberg, 1999), 39.

19. H. Murakami, H. Harada and H.K.D.H. Bhadeshia, "The Location of Atoms in Re- and V-containing Multicomponent Nickel-base Single-Crystal Superalloys," Applied Surf. Sci., 76/77 (1994) 177-183.

20. N. Wanderka and U. Glatzel, "Chemical Composition Measurements of Nickel-base Superalloy by Atom Probe Field Ion Microscopy," Mater. Sci. Eng. A, 203 (1995) 69-74.

21. P.J. Warren, A. Cerezo, and G.D.W. Smith, "An Atom Probe Study of the Distribution of Rhenium in a Nickelbased Superalloy," Mater. Sci. Eng. A, 250 (1998) 8892.

22. J. Rüsing, N. Wanderka, U. Czubayko, V. Naundorf, D. Mukherji, and J. Rösler, "Rhenium Distribution in the Matrix and Near the Particle Matrix Interface in a Model Ni-Al-Ta-Re Superalloy," Scripta Mater., 46 (2002) 235-240.

23. Roger C. Reed, The Superalloys - Fundamentals and Applications (Cambridge: Cambridge University Press, 2006), 162.

24. S.S. Babu, M.K. Miller, J.M. Vitek, and S.A. David, "Characterization of the Microstructure Evolution in a Nickel Base Superalloy during Continuous Cooling Conditions," Acta Mater., 49 (2001) 4149-4160.
25. Mohan P. Pathak, R. Balamuralikrishnan and $\mathrm{K}$. Muraleedharan, Unpublished Research, 2007.

\section{Appendix}

$\underline{\text { Pseudocode for Phase Identification }}$

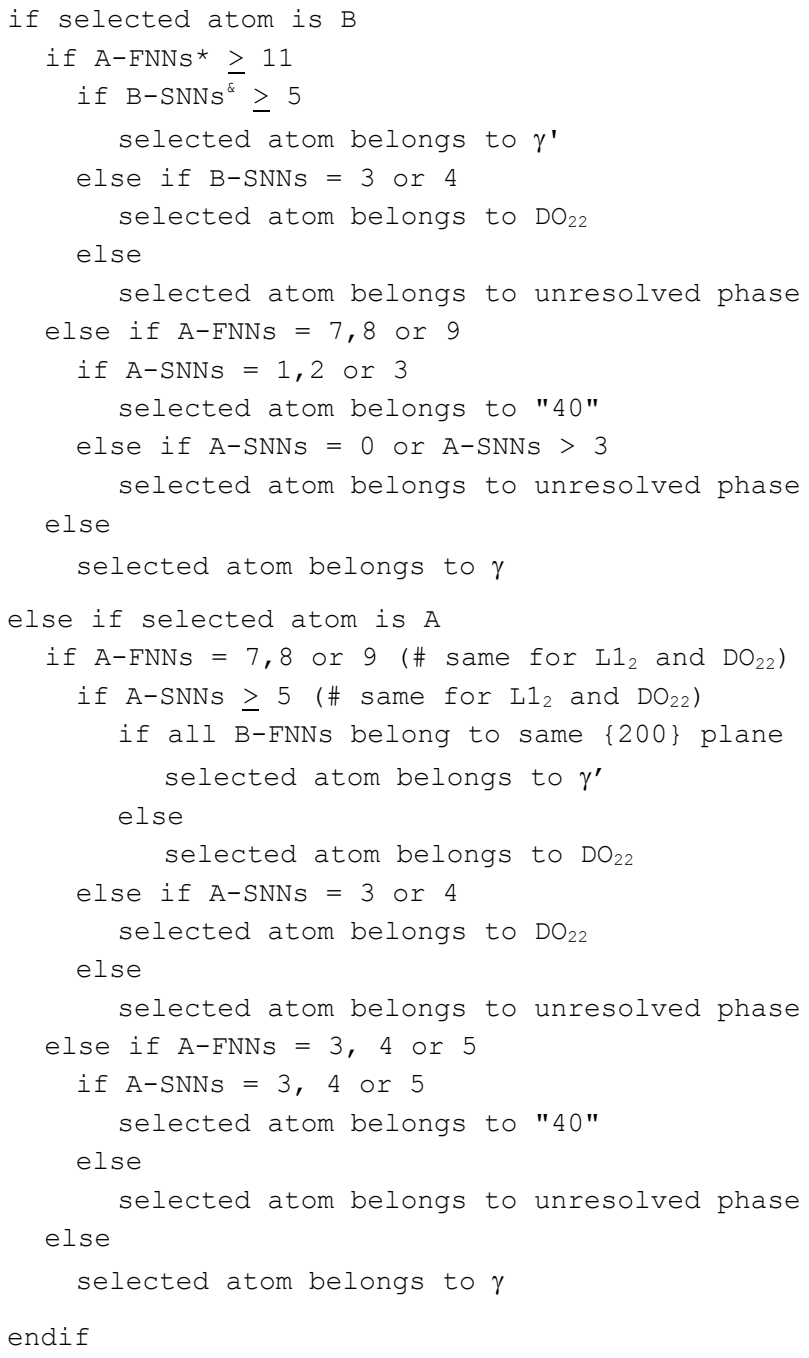

* A-FNNs $\rightarrow$ number of first near neighbor atoms that are A

${ }^{\&} \mathrm{~B}-\mathrm{SNNs} \rightarrow$ number of second near neighbor atoms that are $\mathrm{B}$ 